Conclusion: after $\mathrm{CABG}$, the level of BNP is elevated also in $\mathrm{P}$ with normal ventricular function; the most elevated values were in the immediate proximity of surgery for then being reduced during CR program. The improvement of the diastolic function, pointed out by the increase of the DT, it seems to correlate with the reduction of natriuretic peptides; the relationship of the other indexes of diastolic function, of the EF and of the PAPs with the BNP is detectable only at the second collecting. Is possible to infer that conditions in narrow relationship with the surgery (extracorporeal circulation, quick variations of circulating volume, direct stimulation of the myocardium and impaired lung function due to sternotomy) can induce the liberation of the BNP; this can conceal the association between the incretion of BNP and the indexes of ventricular function.

\section{Improvement in Quadriceps Muscle Strength and Functional Capacities after a 3-Month Home- Based Endurance Training in Lung Transplant Recipients}

Vivodtzev Isabelle, Laboratoire EFCR, CHU de Grenoble \& HP2, Inserm ERI 0017, Université Joseph Fourier, France

Christophe Pison, DMAS. CHU de Grenoble \& LBFA U Inserm 221, Université Joseph Fourier, France

Paulette Mezin, Elisabeth Maclet ${ }^{3}$,

Karen Guerrero, LBFA U Inserm 221, Université Joseph Fourier, France

Jean-Christian Borel, Patrick Levy, Laboratoire EFCR, CHU de Grenoble \& HP2, Inserm ERI 0017, Université Joseph Fourier, France Bernard Wuyam, Laboratoire EFCR, Laboratoire REX-S, Université Joseph Fourier, France

Background: Peripheral muscle dysfunction and reduced exercise capacity are well recognized in lung transplant recipients (LTR) in spite of improved lung function. However, the effect of exercise training in this specific population is still poor studied. We investigated the benefits of training on muscle function and cardio-ventilatory response during exercise in LTR.
Methods: Twelve patients after lung transplantation $\left(\mathrm{FEV}_{1}: 74 \pm 24 \%\right.$ of predicted value) and 12 healthy subjects $(\mathrm{C})$ were studied before and after a 3-month home-based endurance training. Quadriceps strength and fatigability were assessed by maximal twitch tension (TwQ) using magnetic stimulation of the femoral nerve and fiber diameters were obtained after biopsy of the vastus lateralis. Cardio-ventilatory responses were measured during both maximal and constant workload (W) cycle exercise and quality of life was assessed by the Chronic Respiratory Questionnaire.

Results: At baseline, LTR had lower percentage of type I fibers, TwQ, Wmax, $\mathrm{VO}_{2} \max$, VEmax and endurance time than $\mathrm{C}(<50 \%$ of $\mathrm{C}$ each). After training, significant improvement in TwQ $(+44 \pm 26 \mathrm{~N})$, Wmax $(+13 \pm 19 \mathrm{~W})$, endurance time $(+11.5 \pm 11.1$ min) and dyspnea score (CRQdyspnea: $+3.1 \pm 4.4$ points) were observed in LTR ( $p<0.05)$. Furthermore, VE was reduced at submaximal (VE at ventilatory threshold: $-4.5 \pm 6.8 \mathrm{~L} / \mathrm{min})$ and constant $(65 \%$ Wmax $)$ exercise $(-6.4 \pm 5.2 \mathrm{~L} / \mathrm{min})(P<0.05)$. Decrease in exercise-induced fatigability correlated with decrease in VE at initial maximal workload in LTR ( $\mathrm{r}$ $=0.72, P=0.06)$ and in LTR $+\mathrm{C}(\mathrm{r}=0.56, P=0.02)$ and increase in TwQ correlated to increase in CRQdyspnea in LTR ( $\mathrm{r}=0.74, P=0.04)$.

Conclusion: Gains in exercise capacity and in quadriceps strength were observed after endurance training in LTR. The latter may have been involved in improving exercise ventilatory response and dyspnea, supporting the benefit of peripheral muscle strength improvement in exercise tolerance in LTR and even the larger field of chronic respiratory diseases. 